\title{
Temporal Three-Dimensional Ontology for Geographical Information Science (GIS)_A Review
}

\author{
Uznir Ujang*, Alias Abdul Rahman \\ 3D GIS Research Lab, Faculty of Geoinformation and Real Estate, Universiti Teknologi Malaysia, Johor Bahru, Malaysia \\ Email: "*mduznir@utm.my
}

Received April 20, 2013; revised May 20, 2013; accepted June 15, 2013

Copyright (C) 2013 Uznir Ujang, Alias Abdul Rahman. This is an open access article distributed under the Creative Commons Attribution License, which permits unrestricted use, distribution, and reproduction in any medium, provided the original work is properly cited.

\begin{abstract}
While the use of three-dimensional (3D) geographical information system (GIS) is becoming in rapid development and being used in various fields such as urban and regional planning, disaster management and planning, mobile navigation and etc., commercial and open source GIS software packages tend to offer 3D-GIS functionalities for their products. On the basis, GIS analysis functions are to provide information with respect to geographical location and by having 3D spatial data as an input, it will give advantages in providing horizontal position information. However, to analyze moving objects (temporal) in 3D seems not an easy task and not fully supported by current GIS platform packages. Previously in two-dimensional (2D) GIS practice, main issue addressed by researchers in managing temporal spatial objects is GIS packages were designed based on hardware and software constraints whereby it should be based on the temporal spatial objects ontology. Nowadays, the trend of managing temporal 3D data is via 3D spatial simulation or animation. This approach will not in assistance for GIS users in conducting spatial queries. Without having a suitable ontology and valid topological data structure for temporal 3D data, it will cause repetitive of temporal data (redundancy) and complications in executing spatial analysis in 3D environment. Therefore this paper focuses on the ontology for managing moving 3D spatial objects (i.e. air pollution, flood). The characteristics of moving objects were reviewed thoroughly by categorizing it based on its different appearances. Moreover, existing methods in managing temporal database were addressed and discussed for its practicalities. Another important aspect in managing temporal 3D objects is the implementation of topological data structures for 3D spatial objects were reviewed. In the last section of this paper it summarized the issues and further ideas towards implementing and managing temporal 3D spatial objects in GIS based on the Geoinformation Ontology (GeO).
\end{abstract}

Keywords: Multidimensional GIS; Temporal GIS; Geoinformation Ontology; 3D GIS; 3D City Modeling

\section{Introduction}

Users now interested in the visualization of three dimensional (3D) objects [1-3]. It can be seen from users demand in 3D based applications $[4,5]$. These as to facilitate the visualization of $3 \mathrm{D}$ objects as it is more realistic than the two-dimensional (2D) display. Undoubtedly that a $3 \mathrm{D}$ view of a building model is more realistic compared to 2D floor plan of a building. Now most of the major cities have their 3D buildings in order to promote tourism and investors for their cities. Yet the direction of these cities is to increase the total number of 3D buildings in each city (Figure 1).

The trends of 3D city model development can be seen

${ }^{*}$ Corresponding author. from the efforts of many [6,7]. However, most of the 3D city models are mainly used for visualization; perceiving the developments that took place in a city or just to look at the interesting architectural shapes of buildings. Although visualization alone is not sufficient for GIS, but the trend of 3D data usage should be taken into account. Definitely based on the trend, 3D data will be an important resource in the near future. Moreover the analysis of 3D data will become more complex.

The basic functions of Geographical Information System (GIS) to provide information with respect to location may be extended to challenging functions. Currently GIS managed to answer questions about WHAT and WHERE conditions. But there is no doubt that users will be more geared to WHAT, WHERE and WHEN scenarios in the future [8]. The "When" could be divided based on the 


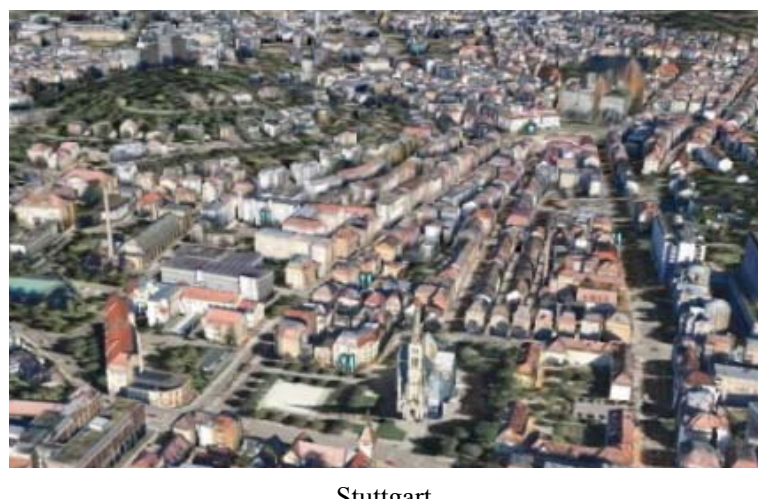

Stuttgart

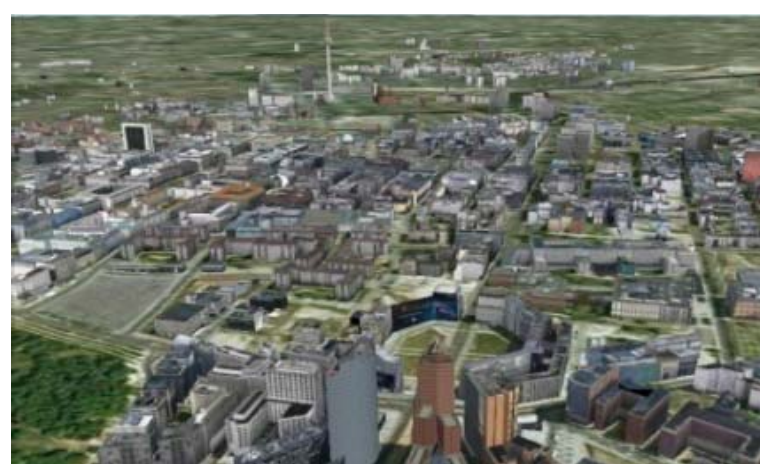

Berlin

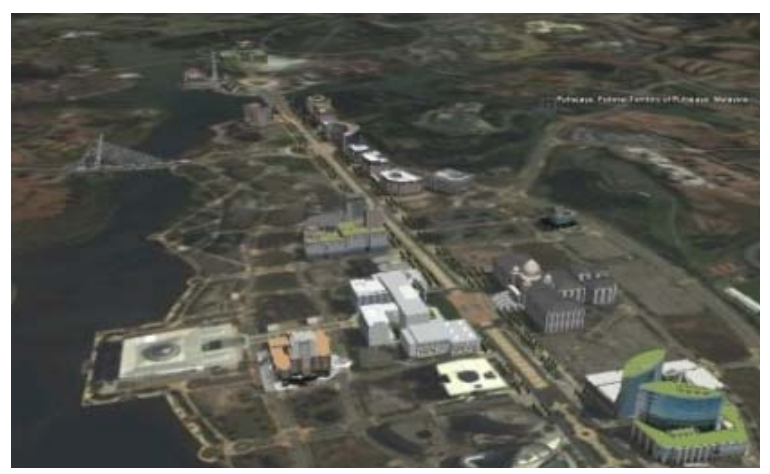

Putrajaya

Figure 1. 3D city models of some major cities (March 2013).

situation whether it had already happened (3D historical information) or when it will happen (3D planning and prediction). For instance a user that conceivably interested in the developments of a city, simple questions will be raised like "when was this new landmark been built and what form of building demolished before this landmark been developed?". More questions raised in more general situations [9]. In this situation, users do not only want to know about the existing 3D data and information, but also about 3D information before it was constructed.

Previous discussions show the need for 3D spatial data management in the future. 3D data will be among the top choice in the future because of its advantages in providing information, which is almost similar to the real world $[10,11]$. Today, $3 \mathrm{D}$ visualization is very useful for plan- ning and monitoring purposes $[12,13]$. Others $3 \mathrm{D}$ aspects such as 3D data management, 3D analysis and 3D data sharing should be taken into account. As for the 3D visualization it is widely implemented. But for the 3D data management, 3D analysis and 3D data sharing needs further study.

Previous section stated "When" in user's queries. It is closely related to the time interval. If there is a time interval/period, then there is a possibility of spatial change. An example, between urban and rural areas, the changes remain in effect. The difference for urban places, changes occur rapidly compared to rural areas due to the developments. Similarly, slopes are classified as dangerous slope and moderate slope, changes are more likely to occur in dangerous slopes in the terrain compared to the moderate. Spatial changes could be in a form of geometry changes, positional changes or attribute feature changes [14]. But other concepts of the classification for spatial changes are based on the concept of geo-atoms [15]. Mentioned before by Nadi and Mahmoud [14] it could be classified as geo-objects. Another classification; geo-fields which stresses the geo-dynamic and static fields.

$3 \mathrm{D}$ objects that change/move in time can be categorized as a moving 3D objects [16]. Erwig, Hartmut, Schneider, and Vazirgiannis [9] stated the integration of space and time is geometries changing over time. Another definition categorized spatial objects that change after certain time interval or period could be classified as dynamic spatial objects [14]. Moving objects in 3D (3D dynamic objects) should be managed intensively. 3D visualization of moving objects currently is mostly displayed by using animations, 3D representation and multimedia approaches. For spatial analysis, 3D data for visualization purposes is not enough. The basic framework for each object need to be studied and different methods should be developed for different characteristics. For example, the phenomenon of urban growth, hydrographic or transportation requires different approaches in the methods of implementation [17]. Important features such as spatial and attribute independently, queries for events and mechanisms for information security should be maintained [18].

To extend GIS to temporal dimension is not an easy task [19]. Researchers have to overcome these issues for future GIS-complex analysis. This is because each object on the earth's surface are moving [20]. The movement could be sluggish or rapid. Records of the spatial objects movement in the 3D can provide advantages in terms of 3D visualization, planning, 3D monitoring and decision-making process [21-23]. Based on historical and present information, these patterns could be recorded and this information is useful to make predictions for future scenarios [24].

In the next section explanation is made on the $3 \mathrm{D}$ 
temporal objects, object characteristic features and how it is in the context of Geoinformation ontology (GeO). Then Section 3 describes the temporal data management in the context of temporal databases, valid and transaction times and temporal database classification. Section 4 discussed about 3D data structure for temporal implementation. At the end of this article discusses and summarization was made for the implementation of 3D temporal objects in $\mathrm{GeO}$.

\section{3D Temporal Objects}

3D temporal objects or moving 3D objects can be classified as objects that involved in two different dimensions. The first dimension is the spatial dimension and the other is the temporal dimension. Spatial dimension is a dimension that linked the position or location of the $3 \mathrm{D}$ object in the real world. While the changes that occur on the 3D object according to a specific time interval designated as the temporal dimension. For spatial dimensions, 2D shows the information about the planar surfaces while the $2.5 \mathrm{D}$ and $3 \mathrm{D}$ shows the complete representation of spatial objects. Furthermore, the 3D data model only describes static topographic conditions [25]. Changes in temporal situation can be viewed from several aspects [26]. Perhaps changes in attribute change over time $(\delta \mathrm{z} /$ $\delta \mathrm{t})$, attributes change over space $(\delta \mathrm{z} / \delta \mathrm{x})$ or spatial change over time $(\delta \mathrm{x} / \delta \mathrm{t})$.

\subsection{Moving 3D Object Characteristics}

Demands for temporal 3D management are increasing [25]. In Euclidean space or Euclidean three-dimensional plane the link between the axes $\mathrm{x}, \mathrm{y}$ and $\mathrm{z}$ were set properly. However, to introduce temporal elements in the axis requires specialized and further research. Goodchild, Yuan and Cova [15] elaborate the commonly observed characteristics of geo-objects through time, based on three conditions (Figure 2).

Object features that are said to be dynamic can be in several forms [27]:

- Moving objects-Objects can be created and destroyed that are free to move and change attributes [26].

- Dynamic fields - Every space-time point has a value for any number of fields.

- Static elastic objects-Objects can be created and destroyed that have dynamic extent and attributes [28].

- Moving elastic objects-Objects can be created, destroyed, move and change extent.

- Moving elastic objects with internal variation-Blend of dynamic fields and objects [15].

- Hybrids-Any combination of the prior user views.

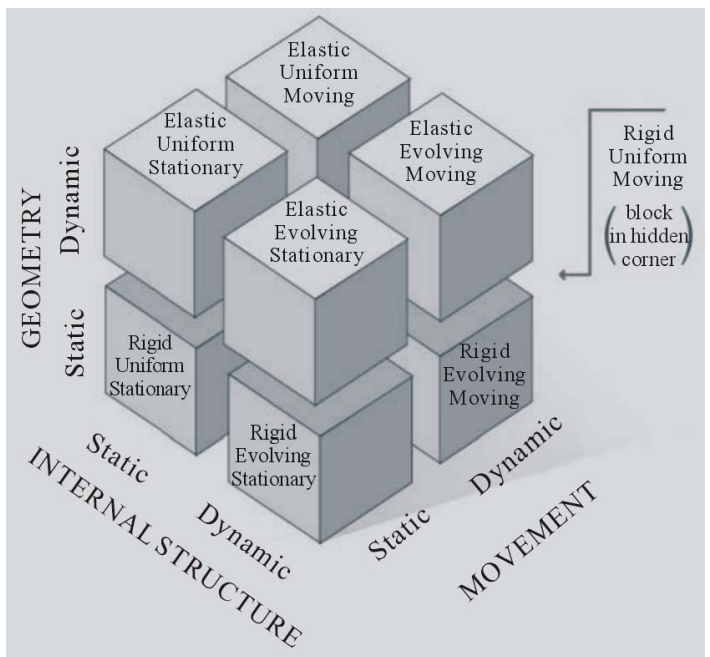

Figure 2. Temporal variability in geo-objects [15].

In ISO, classification of movement has the following characteristics [30]:

- The feature moves within any domain composed of spatial.

- The feature may move along a planned route, but it may deviate from the planned route.

- A motion may be influenced by physical forces, such as orbital, gravitational, or inertial forces.

- The motion of a feature may influence or be influenced by other features, e.g.:

- The moving feature might follow a predefined route (e.g., road) and might change routes at known points (e.g., bus stops, waypoints).

- Two or more moving features may be "pulled" together or pushed apart (e.g., a predator detects and tracks a prey, refugee groups join forces).

- Two or more moving features may be constrained to maintain a given spatial relationship for some period (e.g., tractor and trailer, convoy).

This international standard defines a standard method to describe the geometry of a feature that moves as a rigid body. Transformation of 3D objects (Figure 3), such as Scaling, Translation, Rotation and Reflection in $\mathrm{R}^{3}$ has been translated in the existing $\mathrm{R}^{3}$ matrixes [29]. However, it is only useful for fixed and physical unchanging objects. For other temporal characteristics mentioned, further research is needed for the process of mapping 3D objects in GIS.

Approaches in modelling the 3D temporal objects are through animation, simulation or the method of time stamping [14,31]. The result is satisfactory; just from the aspect of GIS it is only good for visualization purposes. Temporal changes can be in a form of databases, geometry or graphic changes and most methods described are based on the graphic changes. A database structure for 
3D temporal objects is required for GIS analysis. Based on existing approaches mentioned earlier, each animation or simulation of 3D objects must be done separately from the package that is available in GIS. Many applications of geographical information ignore the temporal dimension and the third (vertical) spatial dimension due to GIS technology itself that puts constraints onto these dimensions [15].

\subsection{Temporal 3D Objects in Geo-Information Ontology (GeO)}

Geoinformation ontology $(\mathrm{GeO})$ is imperative from the view of entity classification of geographic information. $\mathrm{GeO}$ process at the beginning of GIS is not well-addressed and the problem arises in determining the data model because of limited to vendors. Now the implementation of ontology in the web has been taken into account [32]. Grenon and Smith [33] also state the Basic Formal Ontology (BFO) in SNAP ontology.

Ontology describes relations between entities. For moving objects, Goodchild, Yuan, and Cova [15] categorize it to rigid and elastic objects (Figure 4). But in the other hand, a procedure to create new 3D objects in the 3D dynamic GIS environment should be taken into account. Yusoff, Uznir Ujang, and Abdul Rahman [34] emphasizes the calculation of 3D volumes in urban runoff and takes into account the movement of water from various water sources and the collision resulted in a new form and eventually lead to increased water volume (Figure 5). It can be seen from the concept of metaball approach [34].

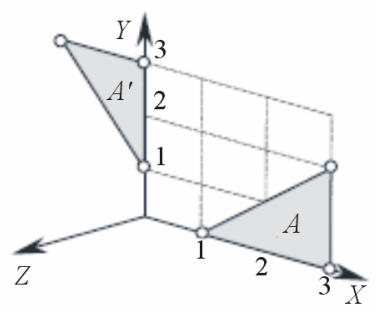

$$
\begin{aligned}
& {\left[\begin{array}{c}
x^{\prime} \\
y^{\prime} \\
z^{\prime} \\
1
\end{array}\right]=\left[\begin{array}{cccc}
a^{2} K+\cos \alpha & a b K-c \sin \alpha & a c K+b \sin \alpha & 0 \\
a b K+c \sin \alpha & b^{2} K+\cos \alpha & b c K-a \sin \alpha & 0 \\
a c K-b \sin \alpha & b c K+a \sin \alpha & c^{2} K+\cos \alpha & 0 \\
0 & 0 & 0 & 1
\end{array}\right] \cdot\left[\begin{array}{l}
x \\
y \\
z \\
1
\end{array}\right]} \\
& {\left[\begin{array}{ccc}
0 & 0 & -2 \\
1 & 3 & 3 \\
0 & 0 & 0 \\
1 & 1 & 1
\end{array}\right]=\left[\begin{array}{cccc}
0 & -1 & 0 & 0 \\
1 & 0 & 0 & 0 \\
0 & 0 & 1 & 0 \\
0 & 0 & 0 & 1
\end{array}\right] \cdot\left[\begin{array}{ccc}
1 & 3 & 3 \\
0 & 0 & 2 \\
0 & 0 & 0 \\
1 & 1 & 1
\end{array}\right]}
\end{aligned}
$$

Figure 3. Arbitrary axis rotation in $R^{3}$ [29].

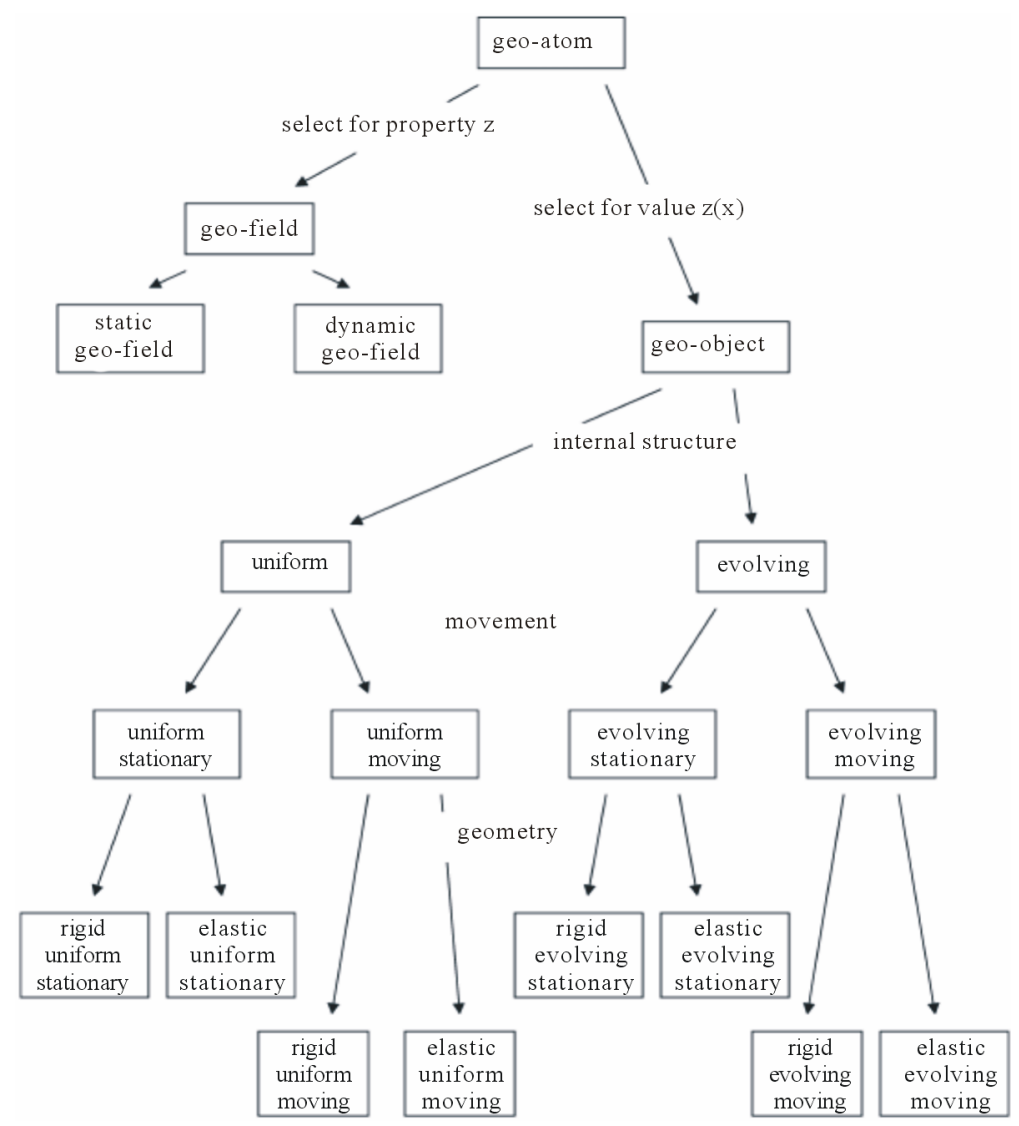

Figure 4. Basic elements of the theory, eight types of dynamic geo-objects [15]. 


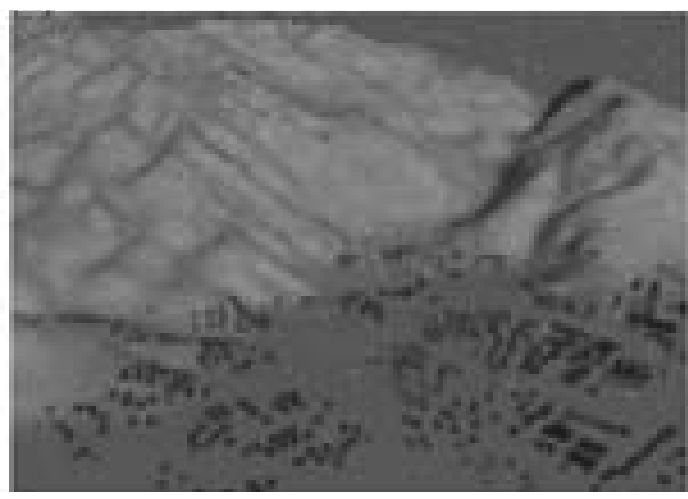

(a)

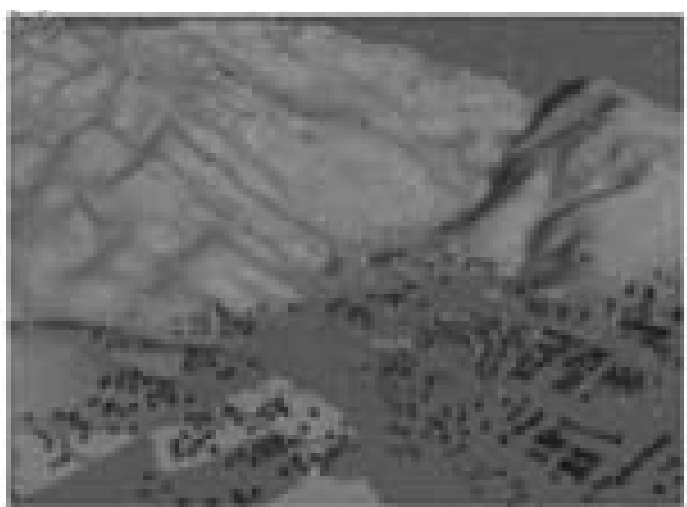

(c)

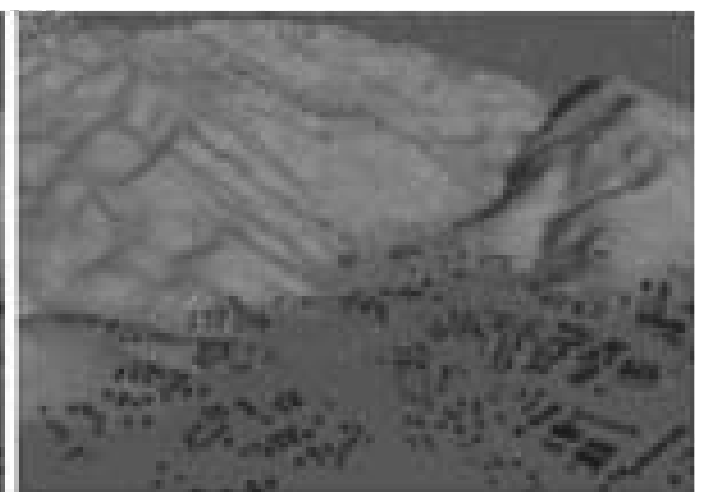

(b)

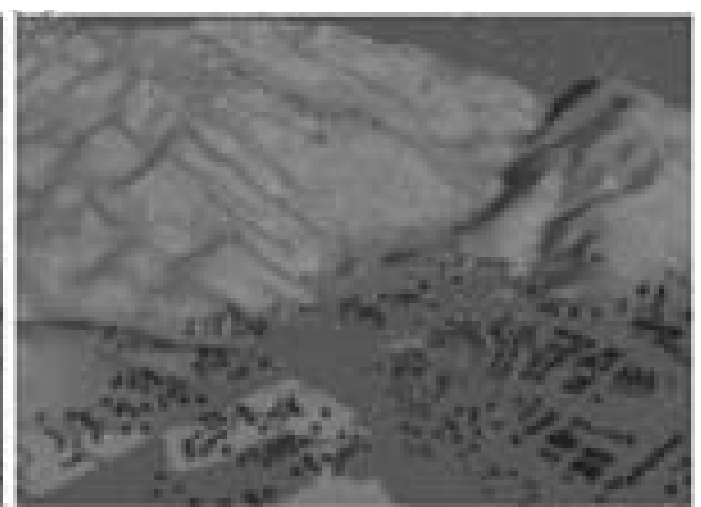

(d)

Figure 5. 3D dynamic VSG modelling for overland flow and open channel flow visualized at (a) 1 hour; (b) 6 hours; (c) 12 hours; (d) 18 hours rainfall data [34].

\section{Temporal Data Management}

\subsection{Temporal Database}

Dynamic object is an object that changed in the interval of time. The time interval requires management of the temporal element. In this section we introduce the main concepts of temporal databases. Then some explanation of the two main time dimensions considered in this area: valid and transaction times. The storage and management of information that has several temporal aspects have been extensively investigated within the research area of temporal databases $[35,36]$. The study of temporal dimensions of data started with much work on the definition of temporally-oriented extensions of the relational model.

\subsection{Valid and Transaction Time}

There are two basic classifications in the temporal dimension. The first one, called transaction time, refers to the moments at which a fact is entered into or deleted from the database; the second one, called valid time, related to the time at which the fact is/was true in the modeled reality. Valid and transaction times are widely recognized as the two basic temporal dimensions of tempo- ral databases [37]. Furthermore, there exists a consolidated terminology about temporal databases dealing with valid and/or the transaction times [38]. Valid and transaction times are defined as: Valid time: The valid time (VT) of a fact is the time when the fact is true in the modeled reality and Transaction time: The transaction time (TT) of a fact is the time when the fact is current in the database and may be retrieved. Valid time is usually provided by the database users, while transaction time is system-generated and supplied.

\subsection{Temporal Database Classification}

A widely accepted classification of valid and transaction times has been proposed in the literature in the past years $[39,40]$. Based on the classification, there are classes that possibly support temporal dimension data. Snapshot databases represent only the current state of the modelled world. It can be achieved by using time specified by the user in the temporal dimension of the database. The database system does not support any information about time semantics, but only the time domain.

Valid-time databases are like historical databases. It supports only the valid time and the history of database updates is not managed. For example, data insertions and 
deletions are not recorded; only the temporal dimensions of the modelled facts are considered. As an analogy, it can represent only the intervals for temporal events, while it is not possible to keep a trace of when the users or personnel entered into or deleted the data from the database.

On the other hand, in transaction-time databases, only the transaction time is supported: users can keep trace of the update operations performed on the database, but not able to represent the temporal dimensions of the represented facts. From previous example, transaction-time databases allow only the times at which the users or personnel enter or deletes the data: the system is not able to support the representation of the intervals during the temporal events.

Meanwhile the bi-temporal database support both transaction and valid time. The system is able to support both the time during the interval and the time where the users or personnel entered or deleted the database.

Prior to temporal modelling, temporal database selection should be done to meet the objective. Based on previous research, a snapshot-database is the most effortless to implement. However the disadvantage of snapshot database is storage issue. All new events need to be put into the database. Besides, this approach led to data redundancy. Any existing object changes in an event, a new record will be recorded and this will increase the number of records for the same object in the database.

For valid-time and transaction-time database, it depends on the application aims. As described earlier, there are advantages and limitations for both types of databases. If valid time is vital, then the valid-time database can be used. However, for transaction-applications based that involves with the importance of transaction instant, so transaction-time database capable to record all business transactions conducted. However if both criteria are needed in the database, then the appropriate choice is the bi-temporal databases.

\section{3D Data Structure}

$3 \mathrm{D}$ data structure is one of the key elements that need to be highlighted in the implementation of $3 \mathrm{D}$ data for temporal situations. There are a variety of $3 \mathrm{D}$ data structures that are still in the spatial-research stage. These data structures were introduced to resolve existing problems in the data structure. This section will look at some previous research on the structure of $3 \mathrm{D}$ data in a temporal environment. The structure is Voronoi diagram data structure, kinetic 3D Voronoi diagram and Dual HalfEdge data structure.

Mostafavi, Beni, and Gavrilova [41] proposed Voronoi diagram as a very powerful spatial model and tested it in different case studies (i.e. gas simulation and global tides simulation) and it offers several advantages compared to other existing spatial models for spatial dynamic representation and dynamic behavior prediction (Figures 6 and 7). Advantages of the Voronoi diagram for temporal field simulation expressed in this research:

- Voronoi diagram is a very interesting data model that can be easily adapted to the configuration of spatial $2 \mathrm{D}$ and $3 \mathrm{D}$ entities in the space.

- It allows clear definition of the spatial relations between objects both in 2D and in 3D spaces.

- It offers an adaptive underlying mesh and grid for geosimulation purposes.

- The dynamic and kinetic operations of the Voronoi diagram are well adapted for the simulation purposes that increase the efficiency and the interactivity of the simulation system.

Ledoux [42] mentioned the usage of the Free-Lagrange Method (FLM)-based on the Voronoi diagram for the modelling of fluid flow in three dimensions (e.g. the movement of underground water or of pollution plumes in the ocean). This technique requires the kinetic threedimensional Voronoi diagram, which is a Voronoi diagram for which the points are allowed to move freely in space (Figure 8).

However, there are limitations in using kinetic 3D Voronoi diagram. It has several complexities that may limit the application of the Voronoi diagram for the simulation purposes.

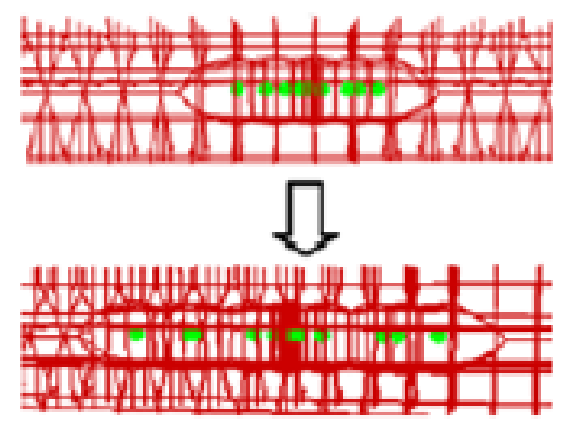

(a)

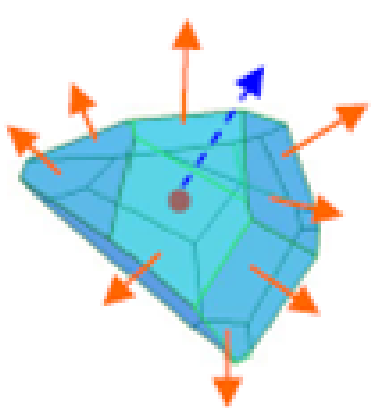

(b)

Figure 6. (a) Simulation of gas dynamics using 3D kinetic Voronoi diagram; (b) Voronoi cell and it's interactions with its neighbours [41]. 


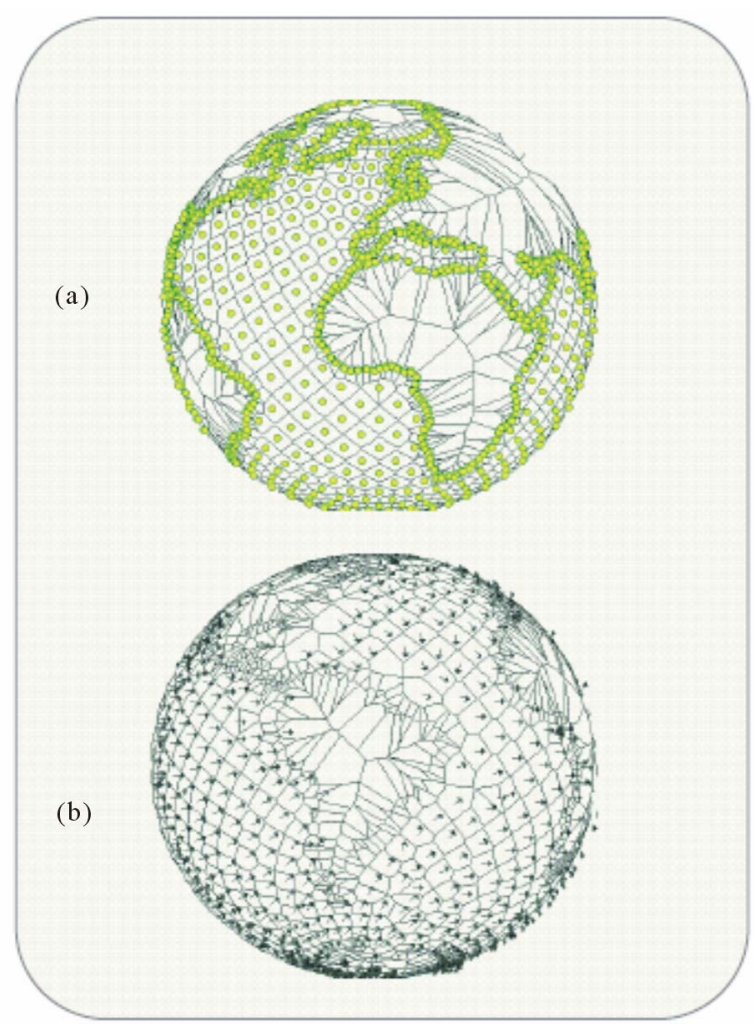

Figure 7. Simulation of global tides using kinetic Voronoi diagram: (a) Initial state; (b) Results after a given simulation time [41].

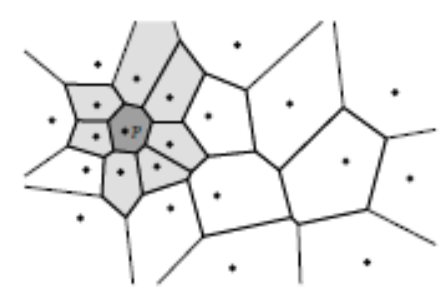

(a)

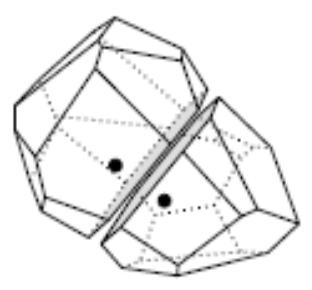

(b)
Figure 8. (a) Voronoi diagram of a set of points in the plane. The point $p$ has seven neighbouring cells; (b) Two Voronoi cells adjacent to each other in $R^{3}$ [42].

- Voronoi diagram has limitation in terms of the complexity of its implementation in the real world. It becomes more difficult when it involves complex 3D spatial objects (i.e. line segments and polygons as well as volumes in 3D space). Architectural designs nowadays are more complex and require accurate modeling for the integrity of the data.

- Mostafavi, Beni, and Hins-Mallet [16] in their paper emphasize that the issues in the implementation of the Voronoi diagram is related to the complex and degeneracy cases related to the implementation of the Voronoi diagram that limits its robustness in some cases.

- Another limitation of the application of the Voronoi diagram is more related to the decartelization of the real world phenomena using the Voronoi diagram. If the spatial and temporal resolutions of the discretization of the dynamic phenomena and the determination of the initial values and boundary conditions are not properly done, then the results of the simulation process may differ and will be unacceptable.

Meanwhile Boguslawski, Gold, and Ledoux [43] presented a new topological data structure (Figure 9), the dual half-edge (DHE), which represent the topology of $3 \mathrm{D}$ buildings and the surrounding terrain. It is based on the idea of simultaneously storing a graph in 3D space and its dual graph.

The DHE uses the half-edges to represent each polyhedron. It contains pairs of half-edges, one in primal space (he) and one in dual space (he.D). Both the primal and dual space are linked together. Other important elements in a basic data structure are pointers. For each half-edge consists its own pointers: to a vertex (he.V); to the paired half-edge that forms the opposite side of the edge (he.S); to the next half-edge around the associated vertex (he. $\mathrm{N}_{\mathrm{V}}$ ); and to the next half-edge around its own face $\left(\right.$ he. $\mathrm{N}_{\mathrm{F}}$ ). The primal part contains a loop pointer around the face of a single cell and the dual part contains a pointer around the face in the dual space. Proposed

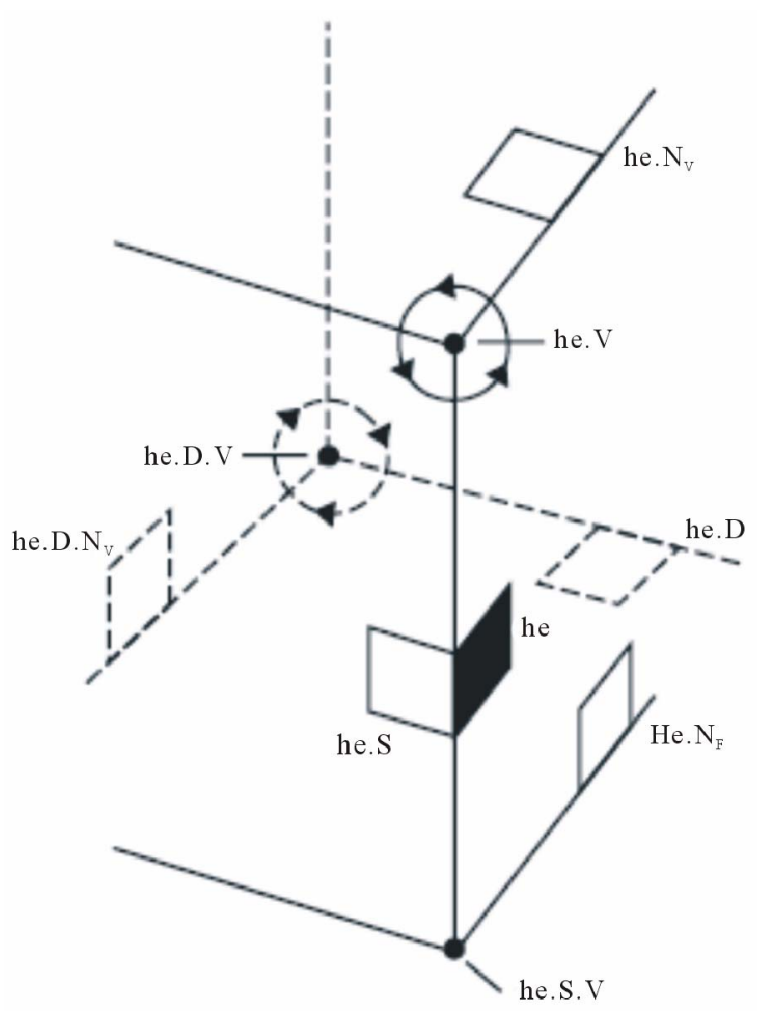

Figure 9. DHE pointer based data structure; primal graph (solid lines) is connected permanently with the dual graph (dashed lines); he-original half-edge; $S, N_{V}, N_{F}, D, V-$ pointers [43]. 
Euler-type operators are for incrementally constructing 3D models for adding individual edges, faces and volumes to the model and updating the dual structure simultaneously. The new data structure is then tested with CityGML models (Figure 10). This technique can manage important query, finding the nearest point (exterior exit to a given room).

There are limitations to these data structures. 3D spatial objects for CityGML typically have a uniform design and relatively easy to make the relationships between the objects involved. Yet when it comes to complex spatial objects such as a combination of manifold objects and non-manifold, a new or improved data structures are required to maintain the object topology relationship. There are several limitations contained in CityGML, but DHE data structure adds a new analytic value to CityGML application.

\section{Discussion and Summary}

Discussions in the previous sections elaborate others research in the scope of 3D GIS, temporal relationship between entities, 3D data structure and topology. In the contexts of temporal 3D objects as discussed in Section 2, approaches in modelling the temporal $3 \mathrm{D}$ objects are through animation, simulation or the method of time stamping. It is based on graphics visualization.

Furthermore, most of the research didn't focus on how the data is represented in the temporal Geoinformation ontology (relationship of dynamic objects as they exist in the real world). The method used is data retrieval from tabular data and then performs graphics simulation. In addition, new data need to be recorded for every occurrence of new events. This resulted in data redundancy plus with the simulation had to be made each time new data is available.

Reviews of existing 3D data structure show most of the data structure focused on maintaining its topological

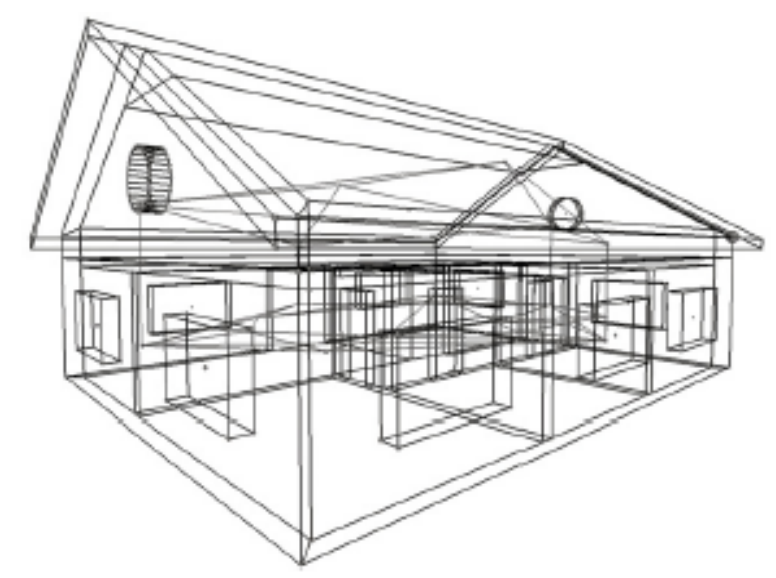

Figure 10. Model of a house reconstructed from the CityGML format using the DHE data structure [43]. relationships between objects (i.e. the topology relationship between edges, vertexes, curves and surfaces). This type of data structure is not sufficient for implementation of a temporal 3D object. A temporal 3D object involves a relationship between entities, such as the geometry of the object and its neighbouring topology. More research should focus on the geometry of the object in the data structure level. In addition, the temporal data should include a temporal element in the database implementation so that events that occurred can be recorded in a welldefined structured database. The selection of the database (valid-time databases, transaction-time database or bitemporal databases) could be validated to see the advantages and limitations in the implementation of 3D spatial database for a temporal object.

Since the real 3D data relationship in the actual world is complex, the data structure should be implemented for 3D complex objects. Current research sees the implementation of data structures based on a uniform object and less study for complex object as well as combinations of uniform objects with complex objects. The relationship between these objects is needed in managing these temporal objects for spatial analysis.

As a conclusion there is a need for further studies in the management of 3D temporal spatial data. As stated by the authors, there are issues from existing scenarios and should be studied. In addition, management of temporal 3D objects is useful in many other fields such as in the fields that require 3D monitoring, 3D planning, disaster management, and other critical application fields.

\section{Acknowledgements}

Major funding for this research was provided by the Ministry of Higher Education Malaysia and partially funded by the Land Surveyors Board of Malaysia.

\section{REFERENCES}

[1] J. Behley and V. Steinhage, "Generation of 3D City Models Using Domain-Specific Information Fusion," In: M. Fritz, B. Schiele and J. H. Piater, Eds., Computer Vision Systems Proceedings, Springer-Verlag, Berlin, 2009, pp. 164-173.

[2] B. Jin and F. Bian, "Study on Visualization of 3D City Model Based on Grid Service," Jisuanji Gongcheng/ Computer Engineering, Vol. 32, No. 4, 2006, pp. 217235.

[3] L. F. Liu and J. Y. Fang, "An Accelerated Approach to Create 3D Web Cities," Ieee, New York, 2009.

[4] M. Freitas, A. A. Sousa and A. Coelho, "Evaluation of Visualization Features in Three-Dimensional LocationBased Mobile Services," Insticc-Inst Syst Technologies Information Control \& Communication, Setubal, 2009.

[5] Z. P. Zhang, J. T. Fang and R. H. Jing, "Interactive Visualisation of 3D City Models Based on Adaptive Stream- 
ing of 3D-GIS Data," Ieee Computer Soc, Los Alamitos, 2009.

[6] M. Over, A. Schilling, S. Neubauer and A. Zipf, "Generating Web-Based 3D City Models from OpenStreetMap: The Current Situation in Germany," Computers, Environment and Urban Systems, Vol. 34, No. 6, 2010, pp. 496507. doi:10.1016/j.compenvurbsys.2010.05.001

[7] A. Toshev, P. Mordohai and B. Taskar, "Detecting and Parsing Architecture at City Scale from Range Data," Proceedings of the Twenty-Third IEEE Conference on Computer Vision and Pattern Recognition, CVPR 2010, San Francisco, 13-18 June 2010, pp. 398-405.

[8] N. Andrienko, G. Andrienko and P. Gatalsky, "Exploratory Spatio-Temporal Visualization: An Analytical Review," Journal of Visual Languages \& Computing, Vol. 14, No. 6, 2003, pp. 503-541. doi:10.1016/S1045-926X(03)00046-6

[9] M. Erwig, R. Hartmut, M. Schneider and M. Vazirgiannis, "Spatio-Temporal Data Types: An Approach to Modeling and Querying Moving Objects in Databases," GeoInformatica, Vol. 3, No. 3, 1999, pp. 269-296. doi:10.1023/A:1009805532638

[10] J. A. Entwistle, K. J. W. McCaffrey and P. W. Abrahams, "Three-Dimensional (3D) Visualisation: The Application of Terrestrial Laser Scanning in the Investigation of Historical Scottish Farming Townships," Journal of Archaeological Science, Vol. 36, No. 3, 2009, pp. 860-866. doi:10.1016/i.jas.2008.11.018

[11] J. O. Lee, G. C. Yi, Y. S. Kim and K. J. We, "3D Wetlands Classification Mapping of Eulsukdo Area Using LiDAR Data," Journal of the Korean Society of Surveying Geodesy Photogrammetry and Cartography, Vol. 27, No. 6, 2009, pp. 639-647.

[12] E. Ayhan, O. Erden and E. Gormus, "Three Dimensional Monitoring of Urban Development by Means of OrthoRectified Aerial Photographs and High-Resolution Satellite Images," Environmental Monitoring and Assessment, Vol. 147, No. 1-3, 2008, pp. 413-421. doi:10.1007/s10661-007-0129-x

[13] Z. Hong, N. Cong, L. Zhang and B. Ai, "3D Monitoring of Ice-Snow in Qinghai-Tibet Plateau-A Case of Geladandong," Second International Conference on Earth Observation for Global Changes, Chengdu, 9 October 2009, pp. 1-8.

[14] S. Nadi and R. D. Mahmoud, "Spatio-Temporal Modeling of Dynamic Phenomena in GIS," ScanGIS'2003-Proceedings of the 9th Scandinavian Research Conference on Geographical Information Science, Espoo, 4-6 June 2003, pp. 302-313.

[15] M. F. Goodchild, M. Yuan and T. J. Cova, "Towards a General Theory of Geographic Representation in GIS," International Journal of Geographical Information Science, Vol. 21, No. 3, 2007, pp. 239-260.

[16] M. A. Mostafavi, L. H. Beni and K. Hins-Mallet, "Representing Dynamic Spatial Processes Using Voronoi Diagrams: Recent Developments," Proceedings of the 2009 Sixth International Symposium on Voronoi Diagrams, Copenhagen, 23-26 June 2009, pp. 109-117.

[17] W. Acevedo and P. Masuoka, "Time-Series Animation
Techniques for Visualizing Urban Growth," Computers \& Geosciences, Vol. 23, No. 4, 1997, pp. 423-435. doi:10.1016/S0098-3004(97)00006-X

[18] S. Nadi and M. R. Delavar, "Toward a General SpatioTemporal Database Structure for GIS Applications," International Symposium on Spatio-Temporal Modeling, Spatial Reasoning, Analysis, Data Mining and Data Fusion, Peking University, Beijing, 2005, pp. 1-3.

[19] W.-J. Gao, J.-X. Zhang, Q. Yan and L. Pang, "Study on Spatio-Temporal Data Model and Visualization Technique," International Symposium on Spatio-temporal Modeling, Spatial Reasoning, Analysis, Data Mining and Data Fusion (ISPRS), Peking University, Beijing, 2005, pp. 1-2.

[20] R. N. Çelik, N. N. Uluğtekin and C. Güney, “4D GeoReferenced Database Approach for GIS," FIG Working Week 2004, Athens, 22-27 May 2004, pp. 1-8.

[21] P.-P. Du, W.-P. Li, S.-X. Sang, L.-X. Wang and X.-Z. Zhou, "Application of 3D Visualization Concept Lay- er Model for Coal-Bed Methane Index System," Procedia Earth and Planetary Science, Vol. 1, No. 1, 2009, pp. 977-981. doi:10.1016/j.proeps.2009.09.151

[22] H. Qu, J. Li and M. Pan, "Three-Dimensional Urban Geological Modeling and Its Applications," 18th International Conference on Geoinformatics, Beijing, 18-20 June, 2010, pp. 1-6.

[23] Z. Xu, X. F. Wu, G. Zhu and H. Yan, "3D Modelling of Groundwater Based on Volume Visualization Technology," In X. Tang, Y. Liu, J. Zhang and W. Kainz, Eds., Advances in Spatio-Temporal Analysis, Taylor \& Francis Group, 2008, pp. 163-168.

[24] X. Liu, W. Liu and J. Hu, "Analysis and Dynamic Simulation of Urban Rainstorm Waterlogging," Sixth International Symposium on Digital Earth: Data Processing and Applications, Beijing, 9-12 September 2009, 7 pp.

[25] A. Abdul-Rahman and M. Pilouk, "Spatial Data Modelling for 3D GIS," Springer, Berlin, Heidelberg, 2008.

[26] C. M. Gold, "Data Structures for Dynamic and Multidimensional GIS," 4th ISPRS Workshop on Dynamic and Multi-Dimensional GIS, Pontypridd, 5-8 September 2005, pp. 36-41.

[27] T. J. Cova, "Dynamic GIS: In Search of the "Killer App," 2006.

http://ncg.nuim.ie/content/events/posters/cova-ngc-2006.p df

[28] N. Käyhkö, N. Fagerholm, B. S. Asseid and A. J. Mzee, "Dynamic Land Use and Land Cover Changes and Their Effect on Forest Resources in a Coastal Village of Matemwe, Zanzibar, Tanzania," Land Use Policy, Vol. 28, No. 1, 2011, pp. 26-37. doi:10.1016/j.landusepol.2010.04.006

[29] J. Vince, "Geometry for Computer Graphics: Formulae, Examples and Proofs," Springer, 2004.

[30] I. O. f. S. ISO, "ISO/TC 211 Geographic Information/ Geomatics," International Organization for Standardization, Switzerland, 2009, pp. 1-98.

[31] A. Stefanidis, K. Eickhorst, P. Agouris and P. Partsinevelos, "Modeling and Comparing Change Using Spatio- 
temporal Helixes," Proceedings of the 11th ACM International Symposium on Advances in Geographic Information Systems, New Orleans, 7-8 November 2003, pp. 8693.

[32] G. Fu, C. Jones and A. Abdelmoty, "Building a Geographical Ontology for Intelligent Spatial Search on the Web," Proceedings of IASTED International Conference on Databases and Applications, Innsbruck, 14-16 February, 2005, pp. 167-172.

[33] P. Grenon and B. Smith, "SNAP and SPAN: Towards Dynamic Spatial Ontology," Spatial Cognition \& Computation: An Interdisciplinary Journal, Vol. 4, No. 1, 2004, pp. 69-104.

[34] I. Mohamad Yusoff, M. Uznir Ujang and A. Abdul Rahman, "3D Volumetric Soft Geo-Objects for Dynamic Urban Runoff Modeling," In: T. Neutens and P. Maeyer, Eds., Developments in $3 D$ Geo-Information Sciences, Springer, Berlin, Heidelberg, 2010, pp. 200-219.

[35] K. Ramamritham, S. H. Son and L. C. DiPippo, "RealTime Databases and Data Services," Real-Time Systems, Vol. 28, No. 2-3, 2004, pp. 179-215. doi:10.1023/B:TIME.0000045317.37980.a5

[36] Y. Wu, S. Jajodia and X. Wang, "Temporal Database Bibliography Update," Temporal Databases: Research and Practice, Vol. 1399, No. 1, 1998, pp. 338-366.

[37] N. Edelweiss, J. de Oliveira and B. Pernici, "An ObjectOriented Approach to a Temporal Query Language," $D a$ - tabase and Expert Systems Applications, Vol. 856, No. 1, 1994, pp. 225-235. doi:10.1007/3-540-58435-8 187

[38] C. S. Jensen and R. T. Snodgrass, "Temporal Projection," Encyclopedia of Database Systems, 2009, pp. 3008-3008.

[39] K. Torp, C. S. Jensen and R. T. Snodgrass, "Effective Timestamping in Databases," The VLDB Journal, Vol. 8, No. 3-4, 2000, pp. 267-288. doi:10.1007/s007780050008

[40] H. Darwen, "Valid Time and Transaction Time Proposals: Language Design Aspects," Temporal Databases: Research and Practice, Vol. 1399, No. 1, 1998, pp. 195210.

[41] M. A. Mostafavi, L. H. Beni and M. Gavrilova, "3D Dynamic Scene Surveillance and Management Using a 3D Kinetic Spatial Data Structure," The International Conference on Advanced Geographic Information Systems \& Web Services, GEOWS 2009, Cancun, 1-7 February 2009, pp. 45-53.

[42] H. Ledoux, "The Kinetic 3D Voronoi Diagram: A Tool for Simulating Environmental Processes," In: P. Oosterom, S. Zlatanova, F. Penninga and E. M. Fendel, Eds., Advances in $3 D$ Geoinformation Systems, Springer, Berlin, Heidelberg, 2008, pp. 361-380.

[43] P. Boguslawski, C. M. Gold and H. Ledoux, "Modelling and Analysing 3D Buildings with a Primal/Dual Data Structure," ISPRS Journal of Photogrammetry and Remote Sensing, Vol. 66, No. 2, 2011, pp. 188-197. doi:10.1016/j.isprsjprs.2010.11.003 